\title{
Mindfulness Based Intervention Program on Anxiety and Depressive Symptoms among Pregnant Women
}

\author{
Sayeda Mohamed Mohamed ${ }^{1}$, Nadia Bassuoni Elsharkawy ${ }^{2}$, Mohamed Hammam Awad ${ }^{3}$ \\ ${ }^{1}$ Department of Psychiatric Mental Health Nursing, Faculty of Nursing, Cairo University, Cairo, Egypt \\ ${ }^{2}$ Department of Maternal and Newborn Health Nursing, Faculty of Nursing, Cairo University, Cairo, Egypt \\ ${ }^{3}$ Department of Obstetrics and Gynaecology Department, Faculty of Medicine, Cairo University, Cairo, Egypt
}

Email address:

Mohamed_sayeda@yahoo.com (S. M. Mohamed), nadiabassuoni@yahoo.com (N. B. Elsharkawy), m_oj_h@yahoo.com (M. H. Awad)

\section{To cite this article:}

Sayeda Mohamed Mohamed, Nadia Bassuoni Elsharkawy, Mohamed Hammam Awad. Mindfulness Based Intervention Program on Anxiety and Depressive Symptoms among Pregnant Women. American Journal of Nursing Science. Vol. 6, No. 3, 2017, pp. $232-239$. doi: 10.11648/j.ajns.20170603.21

Received: March 27, 2017; Accepted: April 8, 2017; Published: April 27, 2017

\begin{abstract}
The aim of this study was to investigate the effect of mindfulness based intervention program on anxiety and depressive symptoms among pregnant women. A Quasi-experimental design was used for this study. This study was conducted at antenatal outpatient clinic at El-Manial Educational University Hospital-Cairo University. A purposive sample consisted of 30 pregnant women with high risk for anxiety and depressive symptoms as confirmed by senior obstetrician according to screening sheet for high risk pregnant women. The sample was divided randomly into two groups; study and control groups (15 each). Three tools were used to collect the data for the present study; a) Personal Data Sheet; b) Edinburgh Postnatal Depression Scale; and c) Perceived Stress Scale. The sample was divided into two groups, fifteen pregnant women as the study group and fifteen pregnant women as control group. Aconstructed mindfulness based intervention program was developed by the researchers and implemented to the study group in ten sessions that were held twice weekly, and each session ranged from 60 to 90 minutes. The main study findings revealed that, there was a statistically significant difference between study and control groups in the reduction of anxiety and depressive symptoms. The study concluded that mindfulness based intervention program was effective with pregnant women concerning the reduction of the anxiety and depression. The study recommended that, there is a great need for performing long-term follow-up in order to evaluate the effects of Mindfulness based intervention program on different pregnancy-related outcomes such as emotional well-being, postnatal depression, and mother-infant relationships.
\end{abstract}

Keywords: Mindfulness, Anxiety, Depression, Pregnant Women

\section{Introduction}

Pregnancy is often portrayed as a time of great joy, that's not the reality for all women. Many women at this is time suffering from confusion, fear, stress, and even depression. American Congress of Obstetricians and Gynecologists (ACOG) [1] reported that, there were $14-23 \%$ of women will struggle with some symptoms of depression during pregnancy. Bennett, et al. [2] found that, the prevalence rates of depression to be $7 \%$ in the first trimester, $13 \%$ in the second trimester, and $12 \%$ in the third trimester. Pregnant women have increased subclinical depressive symptoms, even when potential pregnancy-related confounders such as appetite, somatic symptoms, and sleeping patterns are controlled. Depression is a mood disorder that affects 1 in 4 women at some point during their lifetime, and that illness can affect pregnant women. Depression is not diagnosed properly during pregnancy as it is considered another type of hormonal imbalance [3].

Untreated depression can lead to poor nutrition, drinking, smoking, and suicidal behavior, which can then cause premature birth, low birth weight, and developmental problems. A woman who is depressed often does not have the strength or desire to adequately care for herself or her developing baby. Complications of depression during pregnancy include higher rates of placental abnormalities, preeclampsia, and spontaneous miscarriage [4]. Women can experience depression or anxiety during pregnancy, but women with these risk factors are susceptible; a personal or 
family history of a mood disorder, such as depression or anxiety. Feeling ambivalent about being pregnant and suffer from pregnancy complications. Women may feel more vulnerable and anxious while pregnant and after the birth. Role of obstetrician, maternal and mental health nurses should be considered as they are in direct contact with women who have physiological and psychological health problems during pregnancy. This will provide pregnant women the opportunity to talk about any concerns and to get help if necessary [5].

Anxiety is not only part of being pregnant; it's part of being human. Everyone worries about their lives, and pregnancy can often amplify those worries. Some women are mostly worried about whether their babies are healthy particularly if women have previous pregnancy losses or fertility problems can make this an especially overriding worry. Others might worry about whether they'll be good parents, how their relationships with their partners will change, how siblings will react to a new baby, or the financial aspects of having a child and even if you worry about all these things, that's normal too[6].

There is a strong link between maternal depressive and anxiety symptoms in the third trimester of pregnancy and various fetal developmental problems such as oligohydramnios, IUGR, diminished placental perfusion, and preterm labor [7]. Pharmacological interventions have been well studied for depression unrelated to childbirth; they are not regarded equivocal for depression during pregnancy because of methodological limitations. Only a few studies have examined the efficacy of pharmacological treatments and prevention of depression during pregnancy. There are two main types of antidepressants (ADs) prescribed by physicians to lessen symptoms of Post-partum depression (PPD): serotonin specific re-uptake inhibitors (SSRIs) and tri-cyclic antidepressants [8].

Mindfulness-Based intervention program during pregnancy is considered crucial for successful preventive interventions; for the future wellbeing of the mother, child, and family. It is typically taught in the form of eight to ten weeks' group based educational program focused on the development of mindfulness. Mindfulness is a set of skills such as nonjudgmental awareness of one's present moment experience. While mindfulness practice can induce states of relaxation, it is not a relaxation technique per se as the focus in mindfulness is developing the capacity to simply observe or witness changing mental and physiological states without necessarily trying to alter those states and achieve some desired (e.g. relaxation or calmness) state of mind [9]. Mindfulness-Based intervention program can lessen psychosomatic symptomatology, mood disturbance, medical symptoms, and improve quality of life, \& increase sense of control [10]. Mindfulness practice and yoga have been shown to decrease the perception of pain during labor, reduce physical discomfort during pregnancy and significantly reduce stress, anxiety, and negative emotions [11].

Mindfulness Groups provide a safe setting for members to learn and practice new skills and to receive social support from other members. Groups provide an opportunity for participants to feel less isolated, to be empowered through receiving and offering support, to have their feelings and experiences normalized, and to learn through modeling [12].

\subsection{Significance of the Study}

Pregnancy is generally seen as a time of cheerfulness, fulfillment and joy. Perhaps because of this, depression in pregnancy can be difficult both for women - who often feel guilty about not feeling happy and the people around them to accept and recognize. Antenatal depression can be caused by a hormone imbalance during pregnancy. Other factors that can contribute to antenatal depression are pregnancy sickness and tiredness, anxieties over coping with motherhood and economic and relationship concerns.

Abdalhai and Mosleh [13] studied antepartum anxiety and depression and their association with domestic violence among Egyptian pregnant women. Their results indicated that, pregnant women who expressed simultaneous anxiety and depressive manifestations accounted for $63 \%$, whereas $11.4 \%$ and $10.4 \%$ of them expressed only anxiety and only depression.

Depression and anxiety during pregnancy is a fairly common problem. Pregnant women are either anxious and/or depressed. Plus, mood problems are actually more likely during pregnancy than during the postpartum period. It's difficult to separate the normal symptoms most pregnant women experience - like fatigue, changes in diet, or worry so it can be hard to identify actual symptoms of anxiety and depression.

Antenatal depression can begin at any time during pregnancy and is characterized by having a higher than normal level of worry about the impending birth and parenthood. Symptoms include lack of energy, feeling emotionally detached, tearfulness, chronic anxiety and feeling isolated and guilty. Maternal anxiety and depression have short and long term consequences for women's health, affect mother-child interactions, and increase the risk of a range of ongoing emotional, behavioral and cognitive problems in children.

Non-pharmacological treatments, such as counseling and psychotherapy offer the opportunity to look at the underlying factors that have contributed to depression, as well as helping to change the way you feel. Despite the relative spread of psychosocial interventions that had shown promise for improving outcomes. Research in this area is rather scarce with evidence for success of these therapies remaining controversial. In this work, we provide an evidence based perspective on mindfulness based intervention for healthy pregnancy.

It was found limited studies highlighted the effectiveness of group mindfulness based intervention skills on anxiety and depressive symptoms among pregnant women. So, the researchers conducted this study to make contribution to nursing body of knowledge and obstetrician's role, because antenatal nursing care for pregnant women is essential in caring for those patients. 
The results of this study will be beneficial to obstetrician, maternal and mental health nurses to make programs for prevention and treatment of psychological health problems with pregnant women. Also, it is necessary that, depressive symptoms and anxiety of daily living take an active part in promoting a positive and improvement of psychological health.

\subsection{Aim of the Study}

This study is aimed to investigate the effect of mindfulness based intervention on anxiety and depressive symptoms among pregnant women.

\subsection{Research Hypothesis}

Pregnant women who are subjected to group mindfulness based intervention program will have a higher score than the control who don't receive group mindfulness based intervention program.

\section{Subjects and Methods}

\subsection{Design}

Quasi-experimental design was adopted in this study to achieve the study's aim

\subsection{Setting}

The study was carried out in antenatal outpatient clinic at El-Manial Educational University Hospital - Cairo University, which provides free health care services to pregnant women.

\subsection{Sample}

A purposive sample consisted of 30 pregnant women with high risk for anxiety and depressive symptoms as confirmed by senior obstetrician according to screening sheet for high risk pregnant women.

Women are recruited based on the following inclusion criteria; Eligible subjects from antenatal outpatient clinic who meet criteria were listed alphabetically, then researchers blindly selected the first member from the list, the third name after that are involved in the program till reached 30 pregnant women whose involved-on pretest assessment.

Women were randomly assigned into 2 groups; study group (15 pregnant women) was received group mindfulness based intervention program in addition to the routine antenatal care, and control group(15pregnant women)who receivedroutine antenatal care without group mindfulness based intervention program.

Inclusion criteria

a) Age ranged from 20 to 40 years old.

b) Pregnant women were in second or third trimester.

Additional inclusion criteria were an affirmative response to the question "Have you had a history of mood concerns for which you sought some form of treatment, such as psychotherapy, counselling, or medication?" This method of selecting women based on screening sheet for high risk to depression or anxiety was a result of experience recruiting participants for the initial feasibility group, where finding these pregnant women were reluctant to identify themselves as depressed or anxious, even in the past.

Exclusion criteria:

a) Women had a history of mental disorders as psychosis, dissociation, hallucination, or delusional component.

b) Inability to attend the sessions or participate in the assessments.

\subsection{Tool}

Data was collected through utilizing the following tools:

a) Personal and pregnancy information sheet: It was developed by the researchers to elicit information about pregnant women's age, level of education, and gestational age.

b) Pre/post assessment tools:

a- Edinburgh Postnatal Depression Scale (EPDS) was developed by Cox, Holden \& Sagovsky [14]. The EPDS is easy to administer and has proven to be an effective screening tool. The Edinburgh Postnatal Depression Scale (EPDS) had been previously used for measuring pre-and postnatal depression among women[15]. It consists of ten four-point items. Items 1,2 , and 4 are scored from 0 to 3 while the other items are scored reversely. The total score of the EPDS is $0-30$. Scores of less than 8 were indicated depression not likely, scores 911 were indicatedto depression possible, scores 12-13 were indicated to fairly high possibility of depression and scores of greater than 13 were considered as having depression. The PSS has demonstrated high alpha reliability in pregnant populations [16].

b- Perceived Stress Scale (PSS) was developed by Cohen et al. [17]. PSS is a 14-item measure, which asks participants to rate the extent to which they have felt their life to be stressful within the last month. It measures the degree of self-appraised stress in one's life. It is a fivepoint Likert scale with (1) representing never and (5) representing very often. Sample items from the scale include "In the last month, how often have you been upset because of something that happened unexpectedly?" and "In the last month, how often have you found that you could not cope with all the things that you had to do? Seven items were reversely coded so that higher total scores on the Perceived Stress Scale reflect higher subjective stress. The PSS has a range of scores between 0 and 40. A higher score indicates more stress. Scores around 13 is considered average. High stress groups usually had a stress score of around 20 points. Scores of 20 or higher are considered high stress, may consider learning new stress reduction techniques as well as increasing exercise to at least three times a week. The Perceived Stress Scale has demonstrated good internal consistency and construct validity [18]. In the current study, test-retest reliability for the PSS was 0.85 . The total score of the scale was used to evaluate the level of subjective stress. 


\subsection{The Pilot Study}

A Pilot study was carried out on 3 pregnant women to ensure the clarity and the applicability of the study measures. Nomodifications were needed to test the feasibility of the study tools. Subjects who participated in the pilot study were excluded from the study sample.

\subsection{Ethical Consideration}

Primary permission was attained from the ethical committee of scientific research at the Faculty of Nursing - Cairo University. An official approval to conduct the study was also obtained from both the directors of maternity Hospital and antenatal care clinic. The researchers were contacted pregnant women who met the inclusion criteria of the study. At that time; purpose and nature of the study were explained and informed consent was obtained. Pregnant women have the right to refrain or withdraw from participating in the study at any time without experiencing any negative consequences. Informed consent was obtained from all eligible participants who agreed to participate in the study. Data confidentiality and patients' privacy were secured. Code numbers were created and kept by the researchers.

\subsection{Procedure}

The researchers used a semi structured interview to fill out the tools for pregnant women assessment; this interview lasted for 30-45 minutes. Also, researchers' observations for pregnant women communications and interactions were done. These tools were kept anonymously by using code number. The purpose of the study was explained for pregnant women to gain support and corporations. Fixed time and room was determined for program sessions.

The program was held on 15 sessions twice weekly. The main goals were aimed to teach pregnant women the needed skills to reduce anxiety and improve depressive symptoms during pregnancy. After each session, researchers completed an adherence to treatment form as well as a participant record form in order to maximize intervention fidelity.

Pre-Post tools of assessment were utilized to collect data were explained and filled in individually by the researchers. These pre-assessment questionnaires were completed by all participants (experimental and control groups). After that, the study group was subjected to the mindfulness based intervention developed by the researchers, on base of twice weekly sessions for a period of $60-90$ minutes for each session. At the end of every session, 5-10 minutes were used to review the content of the session and take feedback from the pregnant women. Each session had its own title and objectives according to its content. All steps of the program were carried out by the researchers.

Assessment Phase: This phase was conducted in three sessions (included the study instruments): personal data questionnaire, Perceived Stress Scale, and Edinburgh Postnatal Depression Scale. These pre-assessment questionnaires were completed by all participants (experimental \& control groups) as baseline data.
Implementation Phase: The study group was subjected to the mindfulness based intervention that was developed by the researchers and implemented in twice weekly from $10.00 \mathrm{am}$ - to 11.30 a. m. Sessions. At the end of each session 5-10 minutes were used for conclusion and to get a feedback from the pregnant women.

The contents of each session were as follows:

Session 1. An overview of mindfulness based intervention program, the flow of the program, and the contents of the next sessions. This session focused on establishing trust relationship with pregnant women, and orienting them to the mindfulness based intervention treatment model. The researchers emphasized on confidentiality and maintaining individual's privacy.

Session 2. Resolving minor health problems with pregnant women during second / third trimester.

Session 3. The basic principles of mindfulness

Session 4. Mindful breathing.

Session 5. Step by-step body scanning exercises, and awareness of visceral sensations.

Session 6. Body scanning exercises (continued) and awareness of visceral sensations.

Session 7. Problem solving skill.

Session 8. Interpersonal skill

Session 9: Assertiveness skill

Session 10: Reviewing and planning to use mindfulness skills. At the end of the study participants in control group received training manual of intervention sessions.

Evaluation Phase: It was the final phase of the program. It was conducted in two sessions; post assessment was done by the researchers for all subjects both the study and control groups. This matter helped the researchers to determine the impact of the program on anxiety and depressive symptoms among pregnant women.

\subsection{Statistical Analysis}

The obtained data were reviewed and prepared for computer processing, coded, analyzed and tabulated, using statistical package of the social sciences "SPSS" software version 20. Data was presented using descriptive statistics in the form of frequencies and percentages, means, standard deviations; Correlation coefficient was used to describe association between variables. Inferential statistics t-test and ANOVA (Analysis of Variance) were used. A statistical significance was considered at $\mathrm{P}$ - value $<0.05$.

\section{Results}

This study was conducted on thirty pregnant women to investigate the effect of mindfulness based intervention program on their anxiety and depressive symptoms. Data collection method was utilized and the results of this study were presented as following:

-The age of the studied sample ranged between 20- 40 years, more than one third of the sample $(36.7 \%)$ their ages were 20 25. About one third (33.3\%) of the sample had completed their secondary school education. Sixty percent of the studied sample was in second trimester during the study period. Table 1. 
Table 1. Personal Data of Studied Sample and Pregnancy Information $(n=30)$.

\begin{tabular}{lll}
\hline Personal data & No. & \% \\
\hline Age: & & \\
$20-25$ & 11 & 36.7 \\
$26-30$ & 9 & 30.0 \\
$31-35$ & 9 & 30.0 \\
$36-40$ & 1 & 3.3 \\
Educational level: & & \\
Cannot read and write & 4 & 13.3 \\
Read and Write & 2 & 6.7 \\
Primary school & 8 & 26.7 \\
Secondary school & 10 & 33.3 \\
University & 6 & 20.0 \\
Gestational Age: & & \\
16- 20 & 9 & 30.0 \\
$21-25$ & 9 & 30.0 \\
$26-30$ & 9 & 30.0 \\
$31-35$ & 3 & 10.0 \\
\hline
\end{tabular}

Table 2. Comparison between Mean Scores of Pre and Post assessment of the Experimental and Control Groups Regarding Dependent Studied Variables $(n=30)$.

\begin{tabular}{|c|c|c|c|c|c|}
\hline Variables & Group & Pre Mean \pm SD & Post Mean \pm SD & t-test & $\mathbf{P}$ \\
\hline \multirow{2}{*}{ Stress } & Experimental & $25.5333 \pm 2.85023$ & $19.8000 \pm 2.21037$ & \multirow{2}{*}{-4.920} & \multirow{2}{*}{$0.001 * * *$} \\
\hline & Control & $25.4667 \pm 2.77403$ & $25.000 \pm 2.329993$ & & \\
\hline \multirow{2}{*}{ Depression } & Experimental & $22.3333 \pm 3.01583$ & $10.6667 \pm 2.16025$ & \multirow{2}{*}{-14.569} & \multirow{2}{*}{$0.001 * * *$} \\
\hline & Control & $22.9333 \pm 4.96368$ & $22.2000 \pm 4.31277$ & & \\
\hline
\end{tabular}

Statistically highly significant at $\mathrm{P}<0.001^{*}$

Table 3 showed reduction of stress (ranged between 11-8) and improvement of depression (ranged between 11-7). There was statistically significant relation between pregnant women age groups and perceived stress $(p=0.05)$ without any statistically significant relation between pregnant women age groups and depression. Also, there was no statistically significant difference within different educational level with stress and depression $(p=0.059$ and $p=0.865)$ respectively. Also, there was no statistically significant difference within
-There were significant pre and post program intervention mean scores differences between control and experimental groups regarding dependent studied variables (Cohen perceived Stress and Edinburgh Postnatal Depression Scale). The stress mean scores for post program intervention (experimental group) was significantly different from the mean score for preprogram intervention $(25.5 \pm 2.85,19.8 \pm$ $2.21)$ respectively as compared with control group $(25.5 \pm$ $2.77,25 \pm 2.33$ ). Table 2 .

-Concerning the depression, there were statistically significant differences between mean scores for post program intervention (experimental group) and the mean score for pretest $(22.3 \pm 3.01,10.7 \pm 2.16)$ respectively as compared with control group $(22.9 \pm 4.96,22.2 \pm 4.31)(\mathrm{P}=0.001)$. Table 2.

Table 4. Differences Regarding Age, Levels of Education, and Gestational Age in Experimental Group in Relation to Improvement of Dependent Variables (Cohen perceived Stress and Edinburgh Postnatal Depression Scale).

\begin{tabular}{|c|c|c|c|}
\hline Variables & & Cohen perceived Stress & Edinburgh Post-Natal Depression Scale \\
\hline \multirow{4}{*}{ Age Categories } & $20-25(n=6)$ Mean \pm SD & $18.50 \pm 1.76$ & $10.67 \pm 2.58$ \\
\hline & $26-30(n=5)$ Mean \pm SD & $21.60 \pm 1.51$ & $10.40 \pm 1.67$ \\
\hline & $31-35(n=4)$ Mean \pm SD & $19.50 \pm 2.38$ & $11.00 \pm 2.58$ \\
\hline & P-value & $0.05 * *$ & 0.929 \\
\hline \multirow{6}{*}{ Level of Education } & Cannot read and write $(n=1)$ & 21 & 9 \\
\hline & Read and Write $(n=1)$ & 21 & 9 \\
\hline & Primary school $(n=6)$ Mean \pm SD & $18.0 \pm 1.26$ & $10.8 \pm 2.48$ \\
\hline & Secondary school $(n=5)$ Mean \pm SD & $20.4 \pm 2.30$ & $10.8 \pm 1.92$ \\
\hline & University $(n=2)$ Mean \pm SD & $22.5 \pm 0.7$ & $11.5 \pm 3.53$ \\
\hline & P-Value & 0.059 & 0.865 \\
\hline \multirow{5}{*}{ Gestational Age (weeks) } & $16-20(n=6)$ Mean \pm SD & $18.7 \pm 1.75$ & $10.5 \pm 2.73$ \\
\hline & $21-25(n=4)$ Mean \pm SD & $19.5 \pm 1.73$ & $10.3 \pm 1.25$ \\
\hline & $26-30(n=3)$ Mean \pm SD & $22.0 \pm 2.00$ & $11.0 \pm 2.00$ \\
\hline & $31-35(n=2)$ Mean \pm SD & $20.5 \pm 3.53$ & $11.5 \pm 3.53$ \\
\hline & P-Value & 0.148 & 0.921 \\
\hline
\end{tabular}

Statistically Significant $\mathrm{P}<0.05 * *$

Table 5 showed the correlation matrix among scores of Cohen perceived Stress and Edinburgh Postnatal Depression different gestational age categories with stress and depression $(p=0.184$ and $p=0.921)$ respectively. Table 4 .

Table 3. Range of Post Assessment Improvement Regarding Dependent Variables of the Experimental Group $(n=15)$.

\begin{tabular}{ll}
\hline Variable & Range \\
\hline Stress: & $11-8$ \\
Depression: & $11-7$ \\
\hline
\end{tabular}


Table 5. Correlation Matrix between Total Mean of experimental group as regarding (Cohen perceived Stress and Edinburgh Postnatal Depression Scale).

\begin{tabular}{|c|c|c|c|}
\hline Variables & & $\begin{array}{l}\text { Cohen perceived } \\
\text { Stress }\end{array}$ & $\begin{array}{l}\text { Edinburgh Postnatal } \\
\text { Depression Scale }\end{array}$ \\
\hline $\begin{array}{l}\text { Edinburgh Postnatal } \\
\text { Depression Scale }\end{array}$ & $\begin{array}{l}\mathrm{r} \\
\mathrm{p}\end{array}$ & $\begin{array}{l}0.239 \\
0.390\end{array}$ & 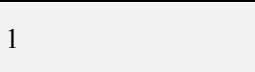 \\
\hline
\end{tabular}

\section{Discussion}

In this research, the researchers attempted to investigate the effect of mindfulness based intervention program on anxiety and depressive symptoms among pregnant women. The findings of this research study supported the research hypotheses which was "Pregnant women who are subjected to group mindfulness based intervention program will have a higher score than the control who don't receive group mindfulness based intervention program".

This research findings indicated that pregnant women who participating in a mindfulness-based intervention have reductions in anxiety and depressive symptoms during pregnancy with statistically significant differences between experimental and control groups (table 2). This could explain that mindfulness based intervention program was effective on reducing participant's anxiety and improve depressive symptoms. A probable reason for lowering anxiety and alleviating of depressive symptoms may be due to the fact that the program has helped the pregnant women to learn new skills to deal with stress and expressed their depressive symptoms in acceptable ways.

Mindfulness skills training can help reduce depression and stress in several ways: First, it teaches the skills necessary for pregnant women to see their mental states as transient not reality. As a result, pregnant women learn how to face the positive and negative content of their thoughts and emotions, which leads to not being drowned to the past and future thoughts. Mindfulness therapies can also facilitate these strategies through acceptance of internal experiences and nonjudgmental awareness to them.

These results were congruent with the study conducted by Yazdanimehr et al. [19] to determine the effect of Mindfulness integrated Cognitive Behavior Therapy on depression and anxiety among pregnant women. They studied 80 pregnant women, 40 in the experimental and 40 in the control groups. The results revealed that of Mindfulness Integrated Cognitive Behavior Therapy was efficient in alleviating depression and anxiety even one month after the intervention.

In the same line, Goodman et al. [20] found that the pregnancy mindfulness-based cognitive therapy intervention has considerable benefits. Effective group interventions are generally more resource and cost-effective than individual therapy. In addition, the group format may provide added therapeutic benefits as indicated by participant feedback regarding the benefits of connecting and learning from other women going through similar experiences.

The results of the current study demonstrated that pregnant women participating in a mindfulness-based intervention showed statistically significant reduction in stress and depression (table 3). This could be explained in light of pregnant women experienced anxiety and depression and need to learn effective strategies to deal with them, this chance provided through mindfulness based intervention program. This finding was consistent with Dimidjian [21] who found that, the mindfulness-based cognitive therapy (MBCT) is a promising method for reducing symptoms of psychological distress and preventing the development of postpartum mood disorders. MBCT tries to get pregnant women to here and now so that they could be detached from the past and future. They discover through observing thoughts that everything is impermanent, and there is no need to worry or fear from thoughts. This detachment helps them to let the thoughts go without magnification of unhelpful thoughts [22].

The current study showed that, there were significant relations between pregnant women's age categories and perceived stress (table 4). This finding could be interpreted as pregnant women were at high risk for anxiety. The treatment program is considered acceptable treatment of anxiety among pregnant women participated and completed the mindfulness based intervention program who reported significant reduction in anxiety. This result was in disagreement with Dayan et al. [23] found that, there were no significant relations between anxiety and the age of pregnant women.

In relation to the differences between levels of education according to the anxiety and depression among pregnant women. The present study revealed that, there was no significant difference between levels of education in the total score of anxiety and depression (table 4). This result was in agreement with Abedian et al. [24] and Podvornik et al. [25] who found that, there were no significant differences in maternal age, educational level, employment status and marital and socio-economic statuses between the respondents in all three pregnancy trimesters. On the other view, this finding was disagreement with Arch [26] revealed that, the pregnant women with a higher education experience less anxiety during pregnancy in comparison to those with lower educational levels

There were not significantly correlated with improvements (change scores) or outcomes. The small sample size made even relatively large correlations no significant, but more importantly, there was no discernible pattern of correlations between attendance and adherence and outcomes.

Regarding to the differences between gestational age according to the anxiety and depression among pregnant women. The present study revealed that, there was no significant difference between gestational age in the total score of anxiety and depression (table 4). This result was not matched with Rezaee and Framarzi [27] who showed that, there was positive correlation between gestational age and pregnancy risk with the depression symptoms.

Concerning the correlation between anxiety and depression among pregnant women (table 5). The present study results revealed that, there were no statistically 
significant correlation between anxiety and depression. The results of the study contributed to the facts that, pregnant women perceived social stigma, and their experience in this regard may have implications not only for how pregnant women are recruited and screened for studies on stress and mood during pregnancy, but also for how pregnant women are screened and assessed for mood problems in clinical settings. This result was consistent with Dayan et al. [23] who did not find any significant correlations between depression and anxiety.

\section{Conclusion}

This study concluded that mindfulness based intervention program was effective approach in reducing anxiety and depression among pregnant women. There was no correlation between the anxiety and depression of the studied sample. One of the strengths of the present study was its focus on improvement of important factors related with anxiety and depression as common problems of pregnant women.

There were not significantly correlated with improvements (change scores) or outcomes. The small samplesize made even relatively large correlations non significant, but more importantly, there was no discerniblepattern of correlations between attendance and adherence and outcomes.

\section{Recommendations}

a) The current study recommended performing long-term follow-up assessments in order to evaluate the effects of mindfulness based intervention program on different pregnancy-related outcomes such as emotional wellbeing, postnatal depression, and mother-infant relationships.

b) Expanding the intervention to bridge between the prenatal and postpartum periods by providing booster sessions postnatally could have value. It remains to be seen what mediates the changes observed, and whether improvements in mood and stress as a result of participating in mindfulness-based interventions will result in improved childbirth and child development outcomes.

c) The early detection and management of depressive and anxiety symptoms during pregnancy should be provided routinely for all pregnant women in Egypt.

d) This study has some limitations. One of the study limitations was related to lack of background knowledge about the study subject matter due to its novelty.

e) Few studies have been conducted so far on these intervention

\section{References}

[1] The American Congress of Obstetricians and Gynecologists (ACOG) (2017).
[2] Bennett, H., Einarson, A., Taddio, A., Koren, G., and Einarson, T. (2004). Prevalence of Depression during Pregnancy: Systematic Review. ObstetGynecol 103: 698-709.

[3] Field, T. (2011). Prenatal Depression Effects on Early Development: A Review. Infant Behav Dev 34:1-14.

[4] Straub H, Adams M, Kim JJ, and Silver, R. K. (2012). Antenatal Depressive Symptoms Increase the likelihood of Preterm Birth. Am J Obstet Gynecol 207:329.e1-329.e4.

[5] National Institute for Health and Care Excellence, (2015). Antenatal and Postnatal Mental Health: Clinical Management and Service Guidance.

[6] Teixeira, C., Figueiredo, F., Conde, A., Pacheco A., and Costa, R. (2009). Anxiety and Depression during Pregnancy in Women and Men. Journal of Affective Disorders Volume 119, Issues 1-3, Pages 142-148.

[7] Sabri,Y., and Nabel, H. (2015). The Impact of Anxiety and Depression during Pregnancy on Fetal Growth and The Birth Outcome. Egypt J Psychiatry Volume: 36, Issue: 2 Page: 95-100.

[8] Canadian Pharmaceutical Association, 2002.

[9] Duncan, L., and Bardacke, N. (2010). Mindfulness- Based Childbirth and Parenting Education: Promoting Family Mindfulness during the Perinatal Period. Journal of Child and Family Studies, 19(2), 190-202.

[10] Vieten, C., and Astin, J. (2008). Effects of a MindfulnessBased Intervention during Pregnancy on Prenatal Stress and Mood: Results of a Pilot Study. Arch Women's Mental Health 11: $67-74$.

[11] Muzik, M., Hamilton, S., Rosenblum, K.,Waxler, E., and Hadi, Z. (2012). Mindfulness Yoga during Pregnancy for Psychiatrically At-Risk Women: Preliminary Results from a Pilot Feasibility Study. Complement Their Clin Pract.; 18(4): 235-240.

[12] Dunn, C., Hanieh, E., Roberts, R., and Powrie, R. (2012). Mindful Pregnancy and Childbirth: Effects of A MindfulnessBased Intervention on Women's Psychological Distress and Well-Being in the Perinatal Period. Archives of Women's Mental Health, 15(2), 139-143.

[13] Abdalhai, R., and Mosleh, H. (2015). Screening for Antepartum Anxiety and Depression and Their Association with Domestic Violence among Egyptian Pregnant Women. J Egypt Public Health Assoc.; 90 (3):101-8.

[14] Cox, L., Holden, M., and Sagovsky, R.(1987). Detection of Postnatal Depression: Development of the 10-Item Edinburgh Postnatal Depression Scale. British Journal of Psychiatry 150:782-786.

[15] Tabrizi M, and Lorestani KH. Effectiveness of the Eclectic Counselling in Decreasing Depression during Pregnancy and Preventing Postnatal Depression. AndishehVaRaftar 2010; 17 (5): 19-26.

[16] Lobel M., DeVincent C. J., Kaminer A., and Meyer, B. A. (2000). The Impact of Prenatal Maternal Stress and Optimistic Disposition on Birth Outcomes in Medically High-Risk Women. Health Psychol 19: 544-553.

[17] Cohen, S., Kamarck, T., and Mermelstein, R. (1983). A Global Measure of Perceived Stress. Journal of Health and Social Behavior, 24, 385-396. 
[18] Roberti, W., Harrington, N., and Storch, A. (2006). Further psychometric support for the 10-item version of the Perceived Stress Scale. Journal of College Counseling, 9(2), 135-147.

[19] Yazdanimehr, R., Omidi, A., Sadat, Z., and Akbari, H. (2016). The Effect of Mindfulness Integrated Cognitive Behavior Therapy on Depression and Anxiety among Pregnant Women: a Randomized Clinical Trial. J Caring Sci. 2016; 5(3):195204.

[20] Goodman, J., Guarino, A., Chenausky, K., Klein, L., Prager, J., Petersen, R., Forget, A., and Freeman, M. (2014). CALM Pregnancy: Results of a Pilot Study of Mindfulness-Based Cognitive Therapy for Perinatal Anxiety. Arch WomensMent Health (2014) 17:373-387.

[21] Dimidjian, S., Goodman, S., Felder, J., Gallop, R., Brown, A., and Beck, A. (2016). Staying well during pregnancy and the postpartum: a pilot randomized trial of mindfulness-based cognitive therapy for the prevention of depressive relapse/recurrence. J Consult Clin Psychol. 84:134-45.

[22] Narimani, M., and Musavi, S. (2014). Effectiveness of Mindfulness Based Cognitive Therapy on Anxiety, Stress and
Depression Of Pregnant Youths: A Randomized Clinical Trial. Eur J Forensic Sci.; Vol 2, Issue 1.

[23] Dayan, J., Creveuil C., Dreyfus, M., Herlicoviez, M, and Baleyte, J. M. (2010). Developmental Model of Depression Applied to Prenatal Depression: Role of Present and Past Life Events, Past Emotional Disorders and Pregnancy Stress. PloS One.; 5:1-8.

[24] Abedian,Z., Soltani,N., Mokhber,N., and Esmaily, H. (2015). Depression and Anxiety in pregnancy and postpartum in women with mild and severe preeclampsia. Iran $\mathrm{J}$ Nurs Midwifery Res. 2015 Jul-Aug; 20(4): 454-459.

[25] Podvornik,N., Elikonja, V., and Praper, P. (2015). Depression and Anxiety in Women during Pregnancy in Solvenia. ZdrVarst.; 54(1): 45-50.

[26] Arch, J. (2013). Pregnancy-specific Anxiety: which Women are Highest and what are the Alcohol-related risks? Compr Psychiatry.; 54:217-28.

[27] Rezaee, R., and Framarzi, M. (2014). Predictors of Mental Health during Pregnancy. Iranian Journal of Nursing and Midwifery Research; 19(7 Suppl1): S45-S50. 